\title{
The pro-inflammatory biomarker soluble urokinase plasminogen activator receptor (suPAR) is associated with incident type 2 diabetes among overweight but not obese individuals with impaired glucose regulation: effect modification by smoking and body weight status
}

\author{
A. Heraclides • T. M. Jensen • S. S. Rasmussen • \\ J. Eugen-Olsen • S. B. Haugaard • K. Borch-Johnsen • \\ A. Sandbak • T. Lauritzen • D. R. Witte
}

Received: 7 February 2013 / Accepted: 3 April 2013 /Published online: 24 April 2013

(C) Springer-Verlag Berlin Heidelberg 2013

\begin{abstract}
Aims/hypothesis Recent evidence links the soluble urokinase plasminogen activator receptor (suPAR), a stable biomarker of systemic immune activation, to several chronic diseases, including type 2 diabetes. suPAR is also associated with adiposity and smoking. We hypothesised that this
\end{abstract}

A. Heraclides · T. M. Jensen • D. R. Witte

Steno Diabetes Center, Gentofte, Denmark

A. Heraclides $(\bowtie)$

The Cyprus Institute of Neurology and Genetics,

International Airport Ave 6,

2370 Nicosia, Cyprus

e-mail: alex.heraclides@gmail.com

\section{S. S. Rasmussen}

Department of Medical Endocrinology, Copenhagen University

Hospital, Rigshospitalet, Copenhagen, Denmark

\section{J. Eugen-Olsen $\cdot$ S. B. Haugaard}

Clinical Research Centre 136, Copenhagen University Hospital, Hvidovre, Denmark

S. B. Haugaard

Department of Internal Medicine,

Copenhagen University Hospital, Amager, Denmark

K. Borch-Johnsen

Research Center for Quality in Health Care, Institute of Public

Health, University of Southern Denmark, Odense, Denmark

A. Sandbæk · T. Lauritzen

Department of Public Health, Section of General Practice,

University of Aarhus, Aarhus, Denmark biomarker would be linked to incident type 2 diabetes in individuals with impaired glucose regulation and that this association would be modified by smoking and body weight status.

Methods The study included 1,933 participants with impaired glucose regulation, who were drawn from the Danish arm of the Anglo-Danish-Dutch Study of Intensive Treatment in People with Screen-Detected Diabetes in Primary Care (ADDITION) and for whom data on suPAR, BMI and smoking were available. Logistic regression analysis was used to estimate the odds for incident type 2 diabetes per twofold increase in suPAR levels. Interactions between both smoking and body weight status and suPAR were tested.

Results During a 3-year follow-up (599 incident diabetes cases), there was a $48 \%$ overall increase in the odds of developing type 2 diabetes per twofold increase in suPAR $(p=0.006)$. This association was modified by body weight status in overweight, but not in obese individuals (OR 2.36, 95\% CI 1.48, 3.76 in overweight group), and by smoking status (OR 2.05, 95\% CI 1.20 , 3.51 in non-smokers). After adjustment for other diabetes risk factors, the association between suPAR and type 2 diabetes was attenuated in the whole sample and among non-smokers, but remained robust among overweight participants.

Conclusions/interpretation suPAR may be a good novel biomarker for systemic sub-clinical inflammation and immune activation linked to incident type 2 diabetes risk in overweight individuals and non-smokers. The observed 
interactions with adiposity and smoking should be investigated further.

Keywords BMI $\cdot$ Body mass index $\cdot$ Impaired glucose regulation · Incident type 2 diabetes $\cdot$ Smoking $\cdot$ Soluble urokinase plasminogen activator receptor $\cdot$ Sub-clinical inflammation $\cdot$ suPAR

\begin{tabular}{|c|c|}
\hline \\
\hline \multirow[t]{3}{*}{ ADDITION } & Anglo-Danish-Dutch Study of Intensive \\
\hline & Treatment in People with Screen-Detected \\
\hline & Diabetes in Primary Care \\
\hline IFG & Impaired fasting glycaemia \\
\hline IGT & Impaired glucose tolerance \\
\hline suPAR & $\begin{array}{l}\text { Soluble urokinase plasminogen activator } \\
\text { receptor }\end{array}$ \\
\hline UPAR & Urokinase plasminogen activator receptor \\
\hline
\end{tabular}

\section{Introduction}

Systemic sub-clinical inflammation and immune activation have been implicated in the pathogenesis of type 2 diabetes [1]. Although the exact causal mechanisms are yet to be determined, inflammatory biomarkers may improve prediction of type 2 diabetes and thus enable early detection, intervention and disease prevention.

The urokinase plasminogen activator receptor (uPAR), which is present in several types of cell, plays a role in numerous physiological pathways mainly involving immune activation, e.g. the plasminogen activating pathway, the modulation of cell adhesion, migration and proliferation, and tissue remodelling and systemic inflammation [2-4]. uPAR is cleaved and released from cells in response to inflammatory stimulation, generating soluble uPAR (suPAR), which can hence be regarded as a pro-inflammatory marker, thus providing an interesting candidate risk biomarker for type 2 diabetes. A few studies have shown that, after adjustment for conventional risk factors, increased levels of suPAR in the general population are associated with an increased risk of death from all causes, as well as increased risk of several chronic diseases, including cancer, cardiovascular disease and type 2 diabetes [5-7].

Existing evidence also suggests that suPAR levels are strongly influenced by adiposity [8] and smoking [5]. However, no evidence exists on the role of body weight status and smoking as effect-modifying factors in the association between suPAR and type 2 diabetes, especially among highrisk individuals.

We aimed to answer the following questions: (1) is suPAR associated with incident type 2 diabetes in people at high risk? and (2) is this association modified by body weight and smoking status?

\section{Methods}

Setting and population The study population was drawn from the Danish arm (DK) of the Anglo-Danish-Dutch Study of Intensive Treatment in People with ScreenDetected Diabetes in Primary Care (ADDITION), which evaluated a systematic screening for people at high risk of type 2 diabetes conducted among all listed patients aged 40 to 69 years in the 175 participating Danish practices [9]. Blood samples collected at baseline screening (2001 to 2006) were used. Details on the original ADDITION recruitment strategy can be found elsewhere [9]. Of the people who completed the risk score questionnaire $(n=134,016$, response rate $82 \%)$, those who had a high risk score $(n=$ $28,041)$ underwent a step-wise clinical assessment comprising the following: determination of random blood glucose and $\mathrm{HbA}_{1 \mathrm{c}}$, and measurement of fasting blood glucose and an OGTT. The aim was to identify cases of undiagnosed type 2 diabetes, as well as impaired fasting glycaemia (IFG) and impaired glucose tolerance (IGT). Of those thus assessed, 1,933 participants had impaired glucose regulation (isolated IFG, isolated IGT or combined IFG and IGT), as well as valid data on suPAR and the potential confounders/effectmodifiers of interest, and were thus included in the current analysis.

Diabetes ascertainment The incidence of type 2 diabetes was ascertained by an OGTT at 1 and 3 years of followup, in combination with regular reports on glucose measurements at GP surgeries up to December 2008.

suPAR assessment suPAR levels were measured in baseline serum samples using a kit (suPARnostic ELISA; ViroGates, Birkerød, Denmark).

Statistical analysis The association between $\log 2-$ transformed suPAR levels at baseline and incident type 2 diabetes was assessed using logistic regression in a sexand age-adjusted model, and in a multivariate-adjusted model (BMI, waist circumference, systolic blood pressure, plasma triacylglycerol, HDL-cholesterol, smoking status). Departure from linearity was assessed by including a quadratic and cubic term in the model, and by performing a likelihood ratio test $(p>0.30$ for all). Analyses were stratified by body weight status based on BMI (lean, overweight, obese according to the WHO 1999 definition [10]) and by smoking status (never/occasional, ex, current), and interactions were tested using the likelihood ratio test.

Ethics Informed consent was obtained from all participants prior to initiation of the study. The study was approved by the local Ethics Committee and the reported investigations 
were carried out in accordance with the principles of the Declaration of Helsinki as revised in 2000.

\section{Results}

Baseline mean suPAR levels were higher: in (1) women than in men; (2) among individuals with isolated IGT and combined IFG and IGT compared with those with isolated IFG; (3) in obese individuals compared with the overweight and lean; and (4) in current and past smokers compared with occasional and never-smokers (Table 1).

During a 3-year follow-up, 599 cases of incident type 2 diabetes were identified. Adjusting for age and sex, there was a $48 \%$ overall increase in the odds of developing type 2 diabetes per twofold increase in baseline suPAR level ( $p=$ 0.006) (Table 2). We found strong evidence for an interaction effect between body weight status and suPAR on incident type 2 diabetes $(p=0.003$ ), while the suPAR $\times$ smoking interaction was marginally non-significant $(p=0.076)$. The association between suPAR and incident type 2 diabetes was only apparent in overweight (BMI 25 to $<30 \mathrm{~kg} / \mathrm{m}^{2}$ ) participants (OR $2.36,95 \%$ CI 1.48, 3.76 per twofold increase in suPAR levels) and in non-smokers (OR 2.05, 95\% CI 1.20, 3.51). Additional adjustment for waist circumference, systolic blood pressure, blood lipids and smoking status attenuated the overall association between suPAR and type 2 diabetes. After these adjustments, the association in the non-smoking group was reduced to marginally non-significant (OR $1.65,95 \%$ CI $0.95,2.89$ ), but the association in the overweight group remained robust
(OR 2.14, 95\% CI 1.31, 3.51). After repeating the analysis while stratifying by quintiles of BMI instead of the WHO classification, the odds of type 2 diabetes increased substantially by $\log 2$-suPAR above a BMI of $28 \mathrm{~kg} / \mathrm{m}^{2}$, with a drop occurring at BMI $>30 \mathrm{~kg} / \mathrm{m}^{2}$. In cross-classification stratified analysis, the association between suPAR and incident type 2 diabetes was particularly high in participants who were overweight and non-smokers $(n=261)$ (OR 5.15, 95\% CI 1.92, 13.78). It should be noted that given a mean of $3.36 \mathrm{ng} / \mathrm{ml}$ and standard deviation of 1.16 in our sample, a twofold increase in suPAR levels represents a relatively big increase (i.e. from the $\sim 10$ th to the $\sim 90$ th percentile of the distribution).

\section{Discussion}

Soluble UPAR, a pro-inflammatory biomarker that is elevated in several infectious diseases, has previously been found to be linked to increased risk of cancer, cardiovascular disease and type 2 diabetes [5, 7]. Here, we show that, in a sample of Danish middle-aged individuals at high risk of diabetes, higher suPAR levels are linked to higher risk of type 2 diabetes in non-smokers and overweight participants. It should be noted here that although we use the word 'risk' in our interpretation, the ORs presented are expected to be larger than the corresponding relative risks, given the high incidence of type 2 diabetes in our study $(\sim 30 \%)$.

Although this study was conducted in a highly selected population (participants with impaired glucose regulation, including somewhat healthier obese participants and
Table 1 Association between suPAR concentrations at baseline and participant characteristics in 1,933 participants at high risk of diabetes in the ADDITION study DK

(2)

${ }^{\mathrm{a}}$ Median (interquartile range)

\begin{tabular}{llll}
\hline Characteristic & $n$ & suPAR $(\mathrm{ng} / \mathrm{ml})^{\mathrm{a}}$ & $p$ value for association \\
\hline Sex & & & \\
$\quad$ Men & 978 & $3.05(2.54,3.68)$ & \\
Women & 955 & $3.28(2.80,3.91)$ & $<0.001$ \\
Glucose regulation status & & & \\
Isolated IFG & 800 & $3.08(2.55,3.71)$ & \\
Isolated IGT & 652 & $3.20(2.75,3.88)$ & \\
IFG and IGT combined & 481 & $3.25(2.80,3.82)$ & 0.001 \\
Body weight status & & & \\
Lean (BMI $\left.<25 \mathrm{~kg} / \mathrm{m}^{2}\right)$ & 341 & $3.08(2.58,3.87)$ & \\
Overweight $\left(\mathrm{BMI} 25\right.$ to $\left.<30 \mathrm{~kg} / \mathrm{m}^{2}\right)$ & 806 & $3.10(2.54,3.68)$ & \\
Obese $\left(\mathrm{BMI} \geq 30 \mathrm{~kg} / \mathrm{m}^{2}\right)$ & 786 & $3.26(2.82,3.87)$ & $<0.001$ \\
Physical activity $(n=1,878)$ & & & \\
Not active & 1,529 & $3.39(2.79,4.08)$ & \\
Active & 349 & $3.17(2.67,3.81)$ & 0.21 \\
Smoking status & & & \\
Non-smokers & 657 & $2.98(2.56,3.45)$ & \\
Occasional smokers & 53 & $2.94(2.47,3.47)$ & \\
Ex-smokers & 691 & $3.11(2.58,3.69)$ & \\
Current smokers & 532 & $3.60(3.03,4.42)$ & $<0.001$ \\
\hline
\end{tabular}


Table 2 Odds ratios for incident type 2 diabetes per twofold increase in baseline suPAR level among 1,933 participants of the ADDITION study DK

\begin{tabular}{|c|c|c|c|c|}
\hline Participant categories & Cases $(n)$ & Total $(n)$ & $\begin{array}{l}\text { Sex- and age-adjusted } \\
\text { OR }(95 \% \mathrm{CI})\end{array}$ & $\begin{array}{l}\text { Sex- }+ \text { age- }+ \text { diabetes-related } \\
\text { covariates }^{\text {a }} \text { OR }(95 \% \text { CI })\end{array}$ \\
\hline Whole sample & 599 & 1,933 & $1.48(1.12,1.96)$ & $1.19(0.88,1.62)$ \\
\hline \multicolumn{5}{|l|}{ By body weight status ${ }^{\mathrm{b}}$} \\
\hline Lean $\left(\mathrm{BMI}<25 \mathrm{~kg} / \mathrm{m}^{2}\right)$ & 70 & 341 & $0.65(0.31,1.37)$ & $0.61(0.26,1.41)$ \\
\hline Overweight (BMI $25-<30 \mathrm{~kg} / \mathrm{m}^{2}$ ) & 231 & 806 & $2.36(1.48,3.76)$ & $2.14(1.31,3.51)$ \\
\hline Obese (BMI $\geq 30 \mathrm{~kg} / \mathrm{m}^{2}$ ) & 298 & 786 & $1.12(0.73,1.73)$ & $1.00(0.63,1.60)$ \\
\hline \multicolumn{5}{|l|}{ By smoking status ${ }^{\mathrm{b}}$} \\
\hline Non-/occasional smokers & 207 & 710 & $2.05(1.20,3.51)$ & $1.65(0.95,2.89)$ \\
\hline Ex-smokers & 218 & 691 & $1.53(0.94,2.51)$ & $1.34(0.78,2.31)$ \\
\hline Current smokers & 174 & 532 & $1.03(0.62,1.71)$ & $0.82(0.47,1.44)$ \\
\hline
\end{tabular}

For the interaction analysis, occasional smokers $(n=53)$ were combined with non-smokers $(n=657)$ in a single group. The model stratified for smoking status does not include smoking as a covariate

${ }^{a}$ BMI, waist circumference, systolic blood pressure, plasma triacylglycerol, HDL-cholesterol, smoking status. Only waist circumference was used in the models stratified by BMI

${ }^{\mathrm{b}}$ Interaction analysis $p=0.003$ for body weight status and $p=0.076$ for smoking status

unhealthier lean participants due to inclusion by design), our findings are in agreement with previous evidence indicating that suPAR levels are strongly associated with body weight and smoking status. A discrepancy with previous evidence is the non-linear association between body weight status and suPAR, which can be explained by the much higher prevalence of smoking in the lean group of our sample (data not shown).

Our results show that the association between suPAR and incident type 2 diabetes is stronger in the overweight participants and in non-smokers. In an attempt to disentangle the effect-modifying roles of body weight and smoking status, we found that the association between suPAR and type 2 diabetes was even stronger in participants who were overweight and non-smokers, indicating that both factors independently modify this association.

An interaction between suPAR and body weight status in relation to type 2 diabetes is plausible, given that adiposity is associated with an unhealthier metabolic profile, and thus sub-clinical inflammation (of which suPAR is a marker) may constitute an additional pathophysiological burden, further increasing the risk of type 2 diabetes. The 'metabolically healthy obese' phenotype [11] of our participants (i.e. non-diabetic despite being obese) may also be resistant to the pathophysiological burden of systemic inflammation, providing a possible explanation of the association found here between suPAR levels and incident type 2 diabetes in overweight, but not in obese individuals.

With regard to smoking, similar findings indicating that suPAR is a better pro-inflammatory biomarker among nonsmokers than among smokers were reported in the Danish MONICA10 cohort study [7]. This proves that, despite the strong linear association between smoking and suPAR, this biomarker is not in the causal pathway between smoking and type 2 diabetes, and may instead represent a genuine proinflammatory pathway in the pathophysiology of the disease.

The exact pathophysiological mechanisms linking suPAR, and more generally sub-clinical inflammation to type 2 diabetes are still unclear. It is believed that suPAR is produced in sites of inflammation by activated neutrophils, which contribute to the recruitment of monocytes to these sites [4], leading to immune activation and under certain conditions systemic sub-clinical inflammation and atherosclerosis, independently of other known inflammatory biomarkers such as C-reactive protein [8]. From the current results, as well as from previous studies, it is clear that smoking and body weight status influence this proinflammatory biomarker.

In conclusion, we provide evidence that suPAR may be a novel pro-inflammatory risk biomarker for progression to type 2 diabetes in overweight and non-smoking individuals at high risk of the disease. This interaction is in agreement with previous studies, but still needs to be further examined, before it can be used in models for type 2 diabetes prediction.

Acknowledgements We thank all participants of the ADDITION study, and the staff responsible for data collection and preparation at the participating Danish general practices.

Funding The ADDITION study received funding from: the Danish Centre for Evaluation and Health Technology Assessment; the Danish Research Foundation for General Practice; the counties of Aarhus, Copenhagen, Ringkoebing, Ribe and South Jutland; the diabetes fund of the National Board of Health; the Danish Medical Research Council number 22-04-0390; the Danish Diabetes Association; the A.P. Møller Foundation; the Bernhard and Marie Kleins Trust; Novo Nordisk A/S and Novo Nordisk Scandinavia; Astra-Zeneca; Pfizer; Servier; GlaxoSmithKline; and HemoCue. 
Duality of interest statement The ADDITION study was given unrestricted grants from Novo Nordisk A/S, Novo Nordisk Scandinavia AB, Astra-Zeneca, Pfizer, Servier, GlaxoSmithKline and HemoCue. J. Eugen-Olsen is co-founder, shareholder and board member of ViroGates A/S, Denmark, the company that developed the suPARnostic ELISA. J. Eugen-Olsen and S. B. Haugaard have patented an invention relating to suPAR and risk of diseases. Copenhagen University Hospital Hvidovre holds the patent, which is licensed to ViroGates $\mathrm{A} / \mathrm{S}$. ViroGates $\mathrm{A} / \mathrm{S}$ had no role in the study design, data collection, analysis or interpretation of study findings, and/or in the decision to submit the manuscript for publication.

At the time of data analysis, A. Heraclides, T. M. Jensen and D. R Witte were employed by Steno Diabetes Center A/S, a research hospital working in the Danish National Health Service and owned by Novo Nordisk A/S. K. Borch-Johnsen was the Director of Steno Diabetes Center from 2000 until 2010. Steno Diabetes Center receives part of its core funding from unrestricted grants from the Novo Nordisk Foundation and Novo Nordisk A/S. K. Borch-Johnsen, T. M. Jensen and D. R Witte own shares in Novo Nordisk A/S.

Contribution statement All co-authors of the current paper provided substantial contributions to at least one of the following: the conception and design of the study, data acquisition, data analysis and interpretation of data. All co-authors were involved in drafting the manuscript or revising it critically for important intellectual content, and provided their final approval of the version to be published. AH was responsible for the data analysis and results write-up and was the main author of the manuscript. TMJ contributed substantially to the interpretation of results and writing up of the manuscript. SSR, JEO, SBH, KBJ, AS, TL and DRW provided critical revisions to the manuscript and contributed valuable intellectual knowledge both in terms of data analysis and results interpretation. DRW conceptualised the idea for the current analysis and was responsible for the overall supervision of the specific project.

\section{References}

1. Kolb H, Mandrup-Poulsen T (2005) An immune origin of type 2 diabetes? Diabetologia 48:1038-1050

2. Blasi F, Carmeliet P (2002) uPAR: a versatile signalling orchestrator. Nat Rev Mol Cell Biol 3:932-943

3. Thunø M, Macho B, Eugen-Olsen J (2009) suPAR: the molecular crystal ball. Dis Markers 27:157-172

4. Mondino A, Blasi F (2004) uPA and uPAR in fibrinolysis, immunity and pathology. Trends Immunol 25:450-455

5. Eugen-Olsen J, Andersen O, Linneberg A et al (2010) Circulating soluble urokinase plasminogen activator receptor predicts cancer, cardiovascular disease, diabetes and mortality in the general population. J Intern Med 268:296-308

6. Persson M, Engström G, Björkbacka $\mathrm{H}$ et al (2012) Soluble urokinase plasminogen activator receptor in plasma is associated with incidence of CVD. Results from the Malmö Diet and Cancer Study. Atherosclerosis 220:502-505

7. Haugaard SB, Andersen O, Hansen TW et al (2012) The immune marker soluble urokinase plasminogen activator receptor is associated with new-onset diabetes in non-smoking women and men. Diabet Med 29:479-487

8. Lyngbæk S, Sehestedt T, Marott JL et al (2012) CRP and suPAR are differently related to anthropometry and subclinical organ damage. Int J Cardiol. doi:10.1016/j.ijcard.2012.03.040

9. Rasmussen SS, Glümer C, Sandbaek A, Lauritzen T, BorchJohnsen K (2008) Determinants of progression from impaired fasting glucose and impaired glucose tolerance to diabetes in a high-risk screened population: 3 year follow-up in the ADDITION study, Denmark. Diabetologia 51:249-257

10. WHO (2000) Obesity: preventing and managing the global epidemic. Report of a WHO consultation. World Health Organ Tech Rep Ser 894:1-253

11. Primeau V, Coderre L, Karelis AD (2011) Characterizing the profile of obese patients who are metabolically healthy. Int J Obes 35:971-981 